\title{
Differential Impact of Cigarette Smoking on Fracture Risks in Subjective Cognitive Decline and Dementia: A Nationwide Longitudinal Study
}

\author{
Yoo Hyun Um¹, Sheng-Min Wang ${ }^{2}$, Kyung-do Han ${ }^{3}$, Nak-Young Kim², \\ Dong Woo Kang ${ }^{4}$, Hae-Ran $\mathrm{Na}^{2}$, Chang Uk Lee ${ }^{4}$, and Hyun Kook Lim ${ }^{2}$ \\ ${ }^{1}$ Department of Psychiatry, St. Vincent's Hospital, College of Medicine, The Catholic University of Korea, Seoul, Republic of Korea \\ 2Department of Psychiatry, Yeouido St. Mary's Hospital, College of Medicine, The Catholic University of Korea, Seoul, Republic of Korea \\ ${ }^{3}$ Department of Statistics and Actuarial Science, Soongsil University, Seoul, Republic of Korea \\ ${ }^{4}$ Department of Psychiatry, Seoul St. Mary's Hospital, College of Medicine, The Catholic University of Korea, Seoul, Republic of Korea
}

Objective We aimed to explore the differential impact of cigarette smoking on fracture risks in SCD and dementia.

Methods A nationwide population-based cohort study design was used. Out of all the people aged $66(n=1,555,103)$ who went through the National Screening Program from 2009-2014, 968,240 participants with eligible data were included in the study. Time-to-event was calculated as the duration between the NSPTA and fracture incidence. Cox proportional-hazard regression analyses were conducted to evaluate the risk of fractures.

Results Increased risk of all [adjusted hazard ratio $(\mathrm{aHR})=1.184 ; 95 \%$ confidence interval $(\mathrm{CI})=1.184,1.093-1.283$ ], hip (aHR=1.518; 95\% CI=1.168-4.972), vertebral ( $\mathrm{aHR}=1.235$; 95\% $\mathrm{CI}=1.101-1.386)$ fractures were increased in current smokers with more than 20 or more pack years ( $\geq 20$ py) of SCD group, after adjusting for all relevant confounding factors. In dementia group, however, current smokers $\geq 20$ py were at reduced risk of hip fractures ( $\mathrm{aHR}=0.249 ; 95 \% \mathrm{CI}=0.089-0.97$ ).

Conclusion There was a disparate influence of cigarette smoking on the fracture risks in SCD and dementia group. Further studies are warranted to explicate this phenomenon, and personalized preventive measures according to one's cognitive status are imperative, since risk factors of fractures can exert disparate influence on patients at different stage of cognitive trajectory.

Psychiatry Investig 2020;17(8):786-795

Key Words Dementia, Cognition, Smoking, Smoking cessation, Fractures.

\section{INTRODUCTION}

Fractures in patients with cognitive impairment is a crucial clinical issue due to their close association with mortality in the elderly. Indeed, patients with mild cognitive impairment (MCI) and dementia are exposed to higher risk of mortality ${ }^{1}$ and lower rates of functional recovery ${ }^{2,3}$ than their counterparts with normal cognition. Dementia is a well-known risk factor for fractures, ${ }^{4,5}$ particularly vulnerable when comorbid

Received: May 5, 2020 Revised: May 24, 2020

Accepted: June 5, 2020

$\triangle$ Correspondence: Hyun Kook Lim, MD, PhD

Department of Psychiatry, Yeouido St. Mary's Hospital, College of Medicine, The Catholic University of Korea, 10 63-ro, Yeongdeungpo-gu, Seoul 07345, Republic of Korea

Tel: +82-2-3779-1048, Fax: +82-2-780-6577, E-mail: drblues@catholic.ac.kr

(a) This is an Open Access article distributed under the terms of the Creative Commons Attribution Non-Commercial License (https://creativecommons.org/licenses/bync/4.0) which permits unrestricted non-commercial use, distribution, and reproduction in any medium, provided the original work is properly cited. with osteoporosis. ${ }^{6}$ Exact mechanisms for the dementia itself being the risk factor for fractures remain elusive, but impairments of physical function including balance, gait, ${ }^{7}$ and sarcopenia that start from early stages of dementia ${ }^{8}$ are purported culprits. Moreover, increasing evidences accentuate the early detection of cognitive frailty, cognitive impairment combined with physical frailty, to minimize further cognitive decline and disability. ${ }^{9}$

In this regard, with physical functional impairments starting from the incipient stage of dementia, it is clinically important to explore the relationship between fracture risks in the elderly in their earliest stages of cognitive impairment. Subjective cognitive decline (SCD) is a recent concept, defined by a complaint of subjective cognitive impairment without any evidences of objective cognitive dysfunction. ${ }^{10}$ Abundant research has been implemented to understand this phenomenon as a premonitory sign of dementia and its importance 
in the unraveling of dementia trajectory. ${ }^{10,11}$ Among studies regarding the relationship between physical function and SCD, SCD group was positively associated with frequent falls when compared with cognitively normal group in one study, ${ }^{12}$ but the study was subject to recall bias and retrospective design that limited generalizability. SCD was independently associated with frailty ${ }^{13}$ and it predicted future falls and upper limb fractures, ${ }^{14}$ but sample sizes in both studies were small. One well-sized population-based cohort study reported a close association with SCD and increased risk of hip fracture in the elderly, but the study only included white individuals with only one type of fracture. ${ }^{15}$ Indeed, studies on SCD and fracture risks are scarce, not to mention the influence of modifiable lifestyle factors on this indispensable relationship.

Among numerous modifiable lifestyle factors, smoking is a major public health threat, with approximately $20 \%$ of worldwide population being current smokers ${ }^{16}$ Hazardous effects of smoking on increasing fracture risk in the elderly have been replicated previously, with approximately two-fold increase of vertebral and hip fracture risks in elderly women followed up for 10 years. ${ }^{17}$ Another study reported a higher risk, with current smokers in the elderly at risk of hip fractures by four fold, ${ }^{18}$ while a Swedish data reported a 2.5 -fold increase of vertebral fractures in current smokers. ${ }^{19}$ Detrimental effects of smoking on musculoskeletal health has been replicated in previous literatures, ${ }^{20}$ with its harmful effects on calciotropic hormonal dysregulation and interruptions in hormone metabolism which result on deterioration in bone health. ${ }^{21}$

In this study, we aimed to compare differential effects of smoking on increasing the risk of fractures in SCD and dementia population. No studies to date compared the fracture risks between SCD and dementia population, and to the best of our knowledge, this is the first study to compare different types of fracture risks in smokers of SCD and dementia patients with nationally representative retrospective cohort. The following were our hypotheses. First, we hypothesized that there will be different patterns of increased fracture risks between SCD and dementia groups. Second, different smoking status (ex-or current smoker) will exert disparate influence on fracture risks in both groups.

\section{METHODS}

\section{Data source}

The Korean National Health Insurance (KNHI) service invites all South Korean residents who reach the ages of 40 and 66 to participate in the screening program named National Screening Program for Transition Ages (NSPTA), in which each age group is considered as a transitional time to middle age and elderly. ${ }^{22}$ It is a part of the National Health Screening
Program (NHSP) which is provided biannually by the KNHI service. $^{23}$ The NSPTA includes comprehensive questionnaires on lifestyle, medical history, depression, cognitive impairment, fall history and urinary dysfunction are included. ${ }^{22}$ As for fracture history of all participants, those with history of fractures were defined by any visit to hospital with any ICD-10 (the 10th revision of the International Statistical Classification of Diseases and Related Health Problems) fracture code within 5 years before the NSPTA. Compared to the subjects aged 40 , those aged 66 are asked for their subjective experience of cognitive impairment through Prescreening Korean Dementia Screening Questionnaire (KDSQ-P). ${ }^{24}$

\section{Study cohort}

Every subject aged 66 who participated in the NSPTA from 2009 to 2014 were included in the study. Out of 1,555,103 participants of the NSPTA, we excluded those with missing values $(n=96,847)$, those without washout with first lag $(n=$ $26,717)$ and those with SCD score below $4(n=463,299)$. A total of 968,240 participants were included for the analyses. Normal cognition (NC) was defined as subjects with a KDSQ-P score of 0 and no history of prescription of acetylcholinesterase inhibitors (donepezil, rivastigmine, galantamine) or NMDA (N-Methyl-D-asparate) receptor antagonist at the time of participation in the NSPTA SCD was defined as subjects with KDSQ-P scores of 4 or more and no history of prescription of acetylcholinesterase inhibitors or NMDA receptor antagonist at the time of participation in the NSPTA. KDSQ$\mathrm{P}$ is a 5-item self-reported questionnaire on a 3-likert scale $(0$ for no, 1 for yes, sometime and 2 for yes, often). 5 questions are as follows: Item $1=$ Do you think that your memory is worse than your peers/friends? Item $2=$ Do you think your memory is worse than last year? Item $3=$ Does your memory decline impact your important activities/work? Item $4=\mathrm{Do}$ others notice your memory decline? Item $5=$ Do you think that you can no longer function as well as before due to your memory decline? Subjects with the history of prescription of acetylcholinesterase inhibitors or NMDA receptor antagonist with an ICD-10 dementia code (F00, F01, F02, F03, G30, F051, or G311) at the time of NSPTA participation were included in the dementia group. To elaborate further, according to the Korea's reimbursement criteria, patient's objective cognitive decline and functional impairment must be evident, meeting the Mini-mental state examination (MMSE) score of 26 or less and Clinical Dementia Rating (CDR) $\geq 1$ or Global Deterioration Scale $\geq 3$ to receive reimbursement for the prescription of either acetylcholinesterase inhibitors or NMDA receptor antagonist. Every participant was asked about their smoking status with the following question: Have you ever smoked more than 5 packs (100 cigarettes) of cigarettes be- 
fore? The following three answer choices were given: 1) No, 2) Yes, but quit smoking, 3) Yes, currently smoking. In both ex-smokers and current smokers, past smoking history was asked, with detailed queries on duration and daily cigarette consumption. Pack years (py) were calculated by multiplying duration and daily cigarette consumption divided by 20. From the day they received NSPTA, the participants were followed up until the occurrence of incidence of fractures, death, or the last follow-up day (December 31, 2016), whichever came first. This study was approved by the Institutional Review Board

Table 1. Baseline characteristics of the study population with incidence of fractures ( $N=968,540)$

\begin{tabular}{|c|c|c|c|c|}
\hline & $\mathrm{NC}^{*}(\mathrm{~N}=759,874)$ & $\mathrm{SCD}^{\dagger}(\mathrm{N}=195,365)$ & Dementia $^{\ddagger}(\mathrm{N}=13,001)$ & $\mathrm{p}$ value \\
\hline Sex, male, N (\%) & $375,642(49.43)$ & $77,062(39.45)$ & $4,977(38.28)$ & $<0.0001$ \\
\hline Age $($ mean $\pm S D)$ & $66 \pm 0$ & $66 \pm 0$ & $66 \pm 0$ & \\
\hline Weight (kg, mean \pm SD) & $62.02 \pm 9.61$ & $60.67 \pm 9.46$ & $59.48 \pm 9.81$ & $<0.0001$ \\
\hline Height (cm, mean $\pm S D$ ) & $159.5 \pm 8.34$ & $158.1 \pm 8.22$ & $157.18 \pm 8.21$ & $<0.0001$ \\
\hline $\mathrm{BMI}\left(\mathrm{kg} / \mathrm{m}^{2}\right.$, mean $\left.\pm \mathrm{SD}\right)$ & $24.34 \pm 3.04$ & $24.24 \pm 3.06$ & $24.04 \pm 3.28$ & $<0.0001$ \\
\hline Waist Circumference $(\mathrm{cm}$, mean \pm SD) & $83.26 \pm 8.23$ & $82.75 \pm 8.35$ & $82.79 \pm 8.81$ & $<0.0001$ \\
\hline Smoking, N (\%) & & & & $<0.0001$ \\
\hline Never & $530,503(69.81)$ & $138,322(70.8)$ & $9,895(76.11)$ & \\
\hline Ex-smoker & $126,503(16.65)$ & $34,009(17.41)$ & $1,863(14.33)$ & \\
\hline Current smoker & $102,868(13.54)$ & $23,034(11.79)$ & $1,243(9.56)$ & \\
\hline Alcohol drinking, N (\%) & & & & $<0.0001$ \\
\hline No & $546,041(71.86)$ & $142,344(72.86)$ & $11,189(86.06)$ & \\
\hline Mild & $129,458(17.04)$ & $32,546(16.66)$ & $1,208(9.29)$ & \\
\hline Moderate & $48,304(6.36)$ & $11,050(5.66)$ & $316(2.43)$ & \\
\hline Heavy & $36,071(4.75)$ & $9,425(4.82)$ & $288(2.22)$ & \\
\hline Regular exercise, yes, N (\%) & $345,644(45.49)$ & $93,672(47.95)$ & $4,702(36.17)$ & $<0.0001$ \\
\hline Income, low, $\mathrm{N}(\%)$ & $227,950(30)$ & $53,676(27.47)$ & $4,244(32.64)$ & $<0.0001$ \\
\hline Diabetes, yes, N (\%) & $156,508(20.6)$ & $39,794(20.37)$ & $3,472(26.71)$ & $<0.0001$ \\
\hline Hypertension, yes, N (\%) & $413,521(54.42)$ & $101,450(51.93)$ & $7,409(56.99)$ & $<0.0001$ \\
\hline Hyperlipidemia, yes, N (\%) & $281,580(37.06)$ & $76,078(38.94)$ & $5,949(45.76)$ & $<0.0001$ \\
\hline Sum of KDSQ-P score, N (\%) & & & & $<0.0001$ \\
\hline 0 & $759,874(100)$ & $0(0)$ & $4,566(35.12)$ & \\
\hline 1 & $0(0)$ & $0(0)$ & $1,213(9.33)$ & \\
\hline 2 & $0(0)$ & $0(0)$ & $1,206(9.28)$ & \\
\hline 3 & $0(0)$ & $0(0)$ & $942(7.25)$ & \\
\hline 4 & $0(0)$ & $68,161(34.89)$ & $887(6.82)$ & \\
\hline 5 & $0(0)$ & $91,092(46.63)$ & $1,710(13.15)$ & \\
\hline 6 & $0(0)$ & $13,920(7.13)$ & $457(3.52)$ & \\
\hline 7 & $0(0)$ & $8,102(4.15)$ & $340(2.62)$ & \\
\hline 8 & $0(0)$ & $5,354(2.74)$ & $356(2.74)$ & \\
\hline 9 & $0(0)$ & $3,094(1.58)$ & $294(2.26)$ & \\
\hline 10 & $0(0)$ & $5,642(2.89)$ & $1,030(7.92)$ & \\
\hline Fracture history, yes, N (\%) & $51,567(6.79)$ & $16,103(8.24)$ & $1,639(12.61)$ & $<0.0001$ \\
\hline
\end{tabular}

*defined as no history of acetylcholinesterase inhibitors or NMDA receptor antagonist with ICD-10 code of dementia (F00, F01, F02, F03, G30, F051) and KDSQ=0, ${ }^{\dagger}$ defined as no history of acetylcholinesterase inhibitors or NMDA receptor antagonist with ICD-10 code of dementia (F00, F01, F02, F03, G30, F051) and KDSQ $\geq 4$, ‡defined as subjects on acetylcholinesterase inhibitors or NMDA receptor antagonist with ICD-10 code of dementia (F00, F01, F02, F03, G30, F051). NC: normal cognition, SCD: subjective cognitive decline, BMI: body mass index; KDSQ-P: Prescreening Korean Dementia Screening Questionnaire, NMDA: N-methyl-D-aspartate, ICD-10: 10th revision of the International Statistical Classification of Diseases and Related Health Problems, AD: Alzheimer's disease, VD: vascular dementia, SD: standard deviation 
of Yeouido St. Mary's Hospital, Seoul, Korea. Consent from individual subjects were not needed because the study used publicly open, anonymous data.

\section{Outcome variable}

All fractures were defined by treatment with any fracturerelated ICD-10 codes. Hip fractures were defined by ICD-10 codes of hip fractures (S72.0 and S72.1) with 1 hospitalization. Vertebral fractures were defined by ICD-10 codes of vertebral fractures (S22.0, S22.1, S32.0, M48.4, and M48.5) with 2 outpatient clinic visits. As for the definition of other fractures, ICD-10 codes for upper arm fractures (S42.0, S42.2, and S42.3), forearm fractures (S52.5 and S52.6), and lower leg fractures (S82.3, S82.5, and S82.6) with 2 outpatient clinic vis- its were included.

\section{Statistical analysis}

Baseline demographic characteristics and clinical characteristics were compared between the three groups using ANOVA for continuous variables and chi-squared test for categorical variables. Time-to-event was calculated as the duration between the NSPTA and fracture incidence. Cox proportionalhazard regression was conducted to evaluate the risk of fractures. All cox proportional hazard regression models were adjusted for potential confounding factors including sex, income, diabetes mellitus, hypertension, hyperlipidemia, alcohol drinking status, physical activity, body mass index (BMI), and fracture history. For all statistical analysis, we used SAS

Table 2. Risk of all fractures according to smoking status and history

\begin{tabular}{|c|c|c|c|c|c|c|c|}
\hline & $\mathrm{N}$ & $\begin{array}{l}\text { All fractures } \\
\text { (total) }\end{array}$ & Duration* & $\mathrm{IR}^{\dagger}$ & $\begin{array}{c}\text { MODEL } 1 \\
\text { aHR (95\% CI) }\end{array}$ & $\begin{array}{c}\text { MODEL } 2 \\
\text { aHR (95\% CI) }\end{array}$ & $\begin{array}{c}\text { MODEL } 3 \\
\text { aHR (95\% CI) }\end{array}$ \\
\hline \multicolumn{8}{|l|}{ NC } \\
\hline Non & 530,503 & 34,073 & $1,842,451.36$ & 18.4933 & 1 (ref.) & 1 (ref.) & 1 (ref.) \\
\hline Ex & 126,503 & 4,125 & $424,404.43$ & 9.7195 & $0.952(0.916,0.99)$ & $0.964(0.927,1.002)$ & $0.961(0.924,0.999)$ \\
\hline Current & 102,868 & 4,647 & $357,392.86$ & 13.0025 & $1.198(1.156,1.242)$ & $1.156(1.114,1.199)$ & $1.156(1.115,1.2)$ \\
\hline Non & 530,503 & 34,073 & $1,842,451.36$ & 18.4933 & 1 (ref.) & 1 (ref.) & 1 (ref.) \\
\hline $\mathrm{Ex} /<20 \mathrm{py}$ & 60,207 & 1,820 & $199,880.58$ & 9.1054 & $0.879(0.835,0.925)$ & $0.9(0.855,0.948)$ & $0.897(0.851,0.944)$ \\
\hline $\mathrm{Ex} / \geq 20 \mathrm{py}$ & 66,296 & 2,305 & $224,523.85$ & 10.2662 & $1.021(0.973,1.07)$ & $1.02(0.972,1.07)$ & $1.019(0.971,1.069)$ \\
\hline Current/<20 py & 32,885 & 1,628 & $112,345.62$ & 14.402 & $1.204(1.143,1.268)$ & $1.182(1.121,1.245)$ & $1.182(1.122,1.245)$ \\
\hline Current/ $\geq 20$ py & 69,983 & 3,029 & $245,047.24$ & 12.3609 & $1.197(1.147,1.249)$ & $1.143(1.094,1.194)$ & $1.143(1.095,1.194)$ \\
\hline \multicolumn{8}{|l|}{ SCD } \\
\hline Non & 138,322 & 11,810 & $524,236.53$ & 22.528 & 1 (ref.) & 1 (ref.) & 1 (ref.) \\
\hline Ex & 34,009 & 1,517 & $121,343.07$ & 12.5017 & $0.985(0.92,1.055)$ & $0.998(0.932,1.069)$ & $0.998(0.931,1.069)$ \\
\hline Current & 23,034 & 1,446 & $86,279.2$ & 16.7595 & $1.227(1.15,1.31)$ & $1.182(1.105,1.263$ & $1.192(1.115,1.274)$ \\
\hline Non & 138,322 & 11,810 & $524,236.53$ & 22.528 & 1 (ref.) & 1 (ref.) & 1 (ref.) \\
\hline $\mathrm{Ex} /<20$ py & 15,380 & 657 & $53,891.96$ & 12.1911 & $0.93(0.852,1.016)$ & $0.952(0.871,1.04)$ & $0.95(0.87,1.038)$ \\
\hline $\mathrm{Ex} / \geq 20$ py & 18,629 & 860 & $67,451.1$ & 12.75 & $1.038(0.955,1.128)$ & $1.038(0.955,1.128)$ & $1.04(0.956,1.13)$ \\
\hline Current/<20 py & 7,088 & 500 & $26,041.82$ & 19.1999 & $1.229(1.12,1.348)$ & $1.2(1.093,1.318)$ & $1.21(1.102,1.328)$ \\
\hline Current $/ \geq 20$ py & 15,946 & 946 & $60,237.38$ & 15.7045 & $1.231(1.138,1.332)$ & $1.174(1.083,1.272)$ & $1.184(1.093,1.283)$ \\
\hline \multicolumn{8}{|l|}{ Dementia } \\
\hline Non & 9,895 & 833 & $29,764.81$ & 27.9861 & 1 (ref.) & 1 (ref.) & 1 (ref.) \\
\hline Ex & 1,863 & 89 & $5,474.52$ & 16.2571 & $0.852(0.658,1.103)$ & $0.846(0.652,1.097)$ & $0.845(0.651,1.096)$ \\
\hline Current & 1,243 & 72 & $3,560.81$ & 20.2201 & $1.017(0.777,1.331)$ & $0.98(0.745,1.289)$ & $0.982(0.746,1.292)$ \\
\hline Non & 9,895 & 833 & $29,764.81$ & 27.9861 & 1 (ref.) & 1 (ref.) & 1 (ref.) \\
\hline $\mathrm{Ex} /<20$ py & 812 & 42 & $2,347.78$ & 17.8893 & $0.903(0.647,1.262)$ & $0.9(0.644,1.26)$ & $0.881(0.63,1.232)$ \\
\hline $\mathrm{Ex} / \geq 20$ py & 1,051 & 47 & $3,126.74$ & 15.0316 & $0.798(0.575,1.109)$ & $0.788(0.567,1.096)$ & $0.8(0.575,1.114)$ \\
\hline Current $/<20$ py & 527 & 36 & $1,491.28$ & 24.1404 & $1.145(0.809,1.622)$ & $1.123(0.792,1.594)$ & $1.143(0.806,1.622)$ \\
\hline Current $/ \geq 20$ py & 716 & 36 & $2,069.53$ & 17.3952 & $0.903(0.63,1.296)$ & $0.853(0.591,1.233)$ & $0.844(0.585,1.219)$ \\
\hline
\end{tabular}

MODEL 1: Sex. MODEL 2: Sex, income, diabetes mellitus, hypertension, hyperlipidemia, alcohol drinking status, physical exercise, body mass index. MODEL 3: Sex, income, diabetes mellitus, hypertension, hyperlipidemia, alcohol drinking status, physical exercise, body mass index, fracture history. *Person-years, tincidence rate per 1,000. NC: normal cognition, SCD: subjective cognitive decline, py: pack years, aHR: adjusted hazard ratio, CI: confidence interval 
version 9.3 (SAS Institute, Cary, NC, USA) with p-values $<0.05$ considered significant.

\section{RESULTS}

\section{Participant characteristics}

A total of 968,540 participants were included in the study. (Table 1) Among them, 75,874 participants were classified as subjects with normal cognition, and 19,5365 with SCD and 13,001 with dementia. Weight, height, body mass index and waist circumference were highest in the normal cognition group, there was a largest number of ex-smokers in the SCD group while number of current smokers highest in the dementia group. As for the alcohol drinking status, heavy drink- ers were mostly distributed in the SCD group. The SCD group reported yes to regular exercise with the highest number, and those with low income levels were most frequent in the dementia group. Regarding metabolic risk factors, prevalence of diabetes, hypertension, hyperlipidemia was concordantly highest in the dementia group Lastly, the number of those with fracture history was highest in the dementia group.

\section{Risk of all fracture incidence}

Risk of all fracture incidence is demonstrated in (Table 2). According to the cox regression models, in subjects with NC, risk of all fractures was increased in current smokers [adjusted hazard ratio $(\mathrm{aHR})=1.156 ; 95 \%$ confidence interval $(95 \%$ $\mathrm{CI})=1.115-1.2]$, but decreased in ex-smokers $(\mathrm{aHR}=0.961$;

Table 3. Risk of hip fractures according to smoking status and history

\begin{tabular}{|c|c|c|c|c|c|c|c|}
\hline & $\mathrm{N}$ & $\begin{array}{l}\text { Hip fractures } \\
\text { (total) }\end{array}$ & Duration* & $\mathrm{IR}^{\dagger}$ & $\begin{array}{c}\text { MODEL } 1 \\
\text { aHR (95\% CI) }\end{array}$ & $\begin{array}{c}\text { MODEL } 2 \\
\text { aHR (95\% CI) }\end{array}$ & $\begin{array}{c}\text { MODEL } 3 \\
\text { aHR (95\% CI) }\end{array}$ \\
\hline \multicolumn{8}{|l|}{$\mathrm{NC}$} \\
\hline Non & 530,503 & 1,607 & $1,842,451.36$ & 0.87221 & 1 (ref.) & 1 (ref.) & 1 (ref.) \\
\hline Ex & 126,503 & 316 & $424,404.43$ & 0.74457 & $0.932(0.809,1.072)$ & $0.982(0.852,1.132)$ & $0.98(0.85,1.129)$ \\
\hline Current & 102,868 & 425 & $357,392.86$ & 1.18917 & $1.464(1.29,1.66)$ & $1.334(1.172,1.519)$ & $1.335(1.173,1.519)$ \\
\hline Non & 530,503 & 1,607 & $1,842,451.36$ & 0.87221 & 1 (ref.) & 1 (ref.) & 1 (ref.) \\
\hline $\mathrm{Ex} /<20$ py & 60,207 & 131 & $199,880.58$ & 0.65539 & $0.821(0.677,0.994)$ & $0.898(0.741,1.089)$ & $0.894(0.737,1.084)$ \\
\hline $\mathrm{Ex} / \geq 20 \mathrm{py}$ & 66,296 & 185 & $224,523.85$ & 0.82397 & $1.029(0.869,1.218)$ & $1.046(0.882,1.24)$ & $1.045(0.881,1.239)$ \\
\hline Current/<20 py & 32,885 & 141 & $112,345.62$ & 1.25506 & $1.535(1.281,1.84)$ & $1.456(1.213,1.749$ & $1.458(1.215,1.751)$ \\
\hline Current/ $\geq 20$ py & 69,983 & 284 & $245,047.24$ & 1.15896 & $1.429(1.236,1.652)$ & $1.275(1.098,1.48)$ & $1.275(1.099,1.48)$ \\
\hline \multicolumn{8}{|l|}{ SCD } \\
\hline Non & 138,322 & 540 & $524,236.53$ & 1.03007 & 1 (ref.) & 1 (ref.) & 1 (ref.) \\
\hline Ex & 34,009 & 142 & $121,343.07$ & 1.17024 & $1.112(0.878,1.409)$ & $1.19(0.939,1.509)$ & $1.19(0.938,1.509)$ \\
\hline Current & 23,034 & 153 & $86,279.2$ & 1.77331 & $1.666(1.332,2.085)$ & $1.563(1.244,1.963)$ & $1.572(1.251,1.975)$ \\
\hline Non & 138,322 & 540 & $524,236.53$ & 1.03007 & 1 (ref.) & 1 (ref.) & 1 (ref.) \\
\hline $\mathrm{Ex} /<20$ py & 15,380 & 53 & $53,891.96$ & 0.98345 & $0.939(0.685,1.288)$ & $1.024(0.746,1.405)$ & $1.021(0.744,1.401)$ \\
\hline $\mathrm{Ex} / \geq 20$ py & 18,629 & 89 & $6,7451.1$ & 1.31947 & $1.247(0.949,1.637)$ & $1.313(1,1.726)$ & $1.316(1.001,1.73)$ \\
\hline Current $/<20$ py & 7,088 & 49 & $26,041.82$ & 1.88159 & $1.788(1.31,2.44)$ & $1.679(1.228,2.295)$ & $1.692(1.238,2.314)$ \\
\hline Current/ $\geq 20$ py & 15,946 & 104 & $60,237.38$ & 1.7265 & $1.613(1.247,2.085)$ & $1.511(1.163,1.963)$ & $1.518(1.168,1.972)$ \\
\hline \multicolumn{8}{|l|}{ Dementia } \\
\hline Non & 9,895 & 96 & $29,764.81$ & 3.22529 & 1 (ref.) & 1 (ref.) & 1 (ref.) \\
\hline Ex & 1,863 & 14 & $5,474.52$ & 2.5573 & $0.554(0.297,1.035)$ & $0.634(0.338,1.186)$ & $0.632(0.337,1.184)$ \\
\hline Current & 1,243 & 12 & $3,560.81$ & 3.37002 & $0.752(0.391,1.444)$ & $0.716(0.368,1.393)$ & $0.721(0.371,1.404)$ \\
\hline Non & 9,895 & 96 & $29,764.81$ & 3.22529 & 1 (ref.) & 1 (ref.) & 1 (ref.) \\
\hline $\mathrm{Ex} /<20$ py & 812 & 7 & $2,347.78$ & 2.98154 & $0.649(0.289,1.458)$ & $0.748(0.332,1.683)$ & $0.728(0.323,1.639)$ \\
\hline $\mathrm{Ex} / \geq 20 \mathrm{py}$ & 1,051 & 7 & $3,126.74$ & 2.23875 & $0.472(0.209,1.068)$ & $0.53(0.233,1.202)$ & $0.539(0.237,1.223)$ \\
\hline Current/<20 py & 527 & 9 & $1,491.28$ & 6.0351 & $1.38(0.672,2.836)$ & $1.255(0.608,2.593)$ & $1.308(0.633,2.703)$ \\
\hline Current $/ \geq 20$ py & 716 & 3 & $2,069.53$ & 1.4496 & $0.311(0.095,1.011)$ & $0.298(0.09,0.985)$ & $0.294(0.089,0.97)$ \\
\hline
\end{tabular}

MODEL 1: Sex. MODEL 2: Sex, income, diabetes mellitus, hypertension, hyperlipidemia, alcohol drinking status, physical exercise, body mass index. MODEL 3: Sex, income, diabetes mellitus, hypertension, hyperlipidemia, alcohol drinking status, physical exercise, body mass index, fracture history. *Person-years, tincidence rate per 1,000. NC: normal cognition, SCD: subjective cognitive decline, py: pack years, aHR: adjusted hazard ratio, CI: confidence interval 
95\% CI=0.924-0.999). With consideration for the pack years, current smokers with pack years of 20 or more ( $\geq 20$ py) were at higher risk when compared with ex-smokers $\geq 20$ py ( $\mathrm{aHR}=$ 1.143; 95\% CI=1.095-1.194). Concordant results were evident in the SCD group as well, with increased risk in current smokers $\geq 20$ py $(\mathrm{aHR}=1.184 ; 95 \% \mathrm{CI}=1.093-1.283)$. No significant findings were observed in the dementia group.

\section{Risk of hip fracture incidence}

Risk of hip fracture incidence is demonstrated in (Table 3). According to the cox regression models, in subjects with $\mathrm{NC}$, risk of hip fractures was increased only in current smokers $(\mathrm{aHR}=1.335$; 95\% $\mathrm{CI}=1.1723-1.519)$. However, when pack year was taken into account, current smokers $<20$ py, and current smokers $\geq 20$ py were both at increased risk of hip fractures. In SCD group, the results were concordant, with increased risks in current smokers, ex-smokers $\geq 20$ py, current smokers $<20$ py, current smokers $\geq 20$ py, with highest risk noted in current smokers with $<20$ py $(\mathrm{aHR}=1.692 ; 95 \% \mathrm{CI}=$ 1.238-2.314). In contrast with the previous two groups, in dementia group, hip fracture incidence risk was decreased in current smokers $\geq 20$ py ( $a H R=0.844 ; 95 \% \mathrm{CI}=0.585-1.219$ ).

\section{Risk of vertebral fracture incidence}

Risk of vertebral fracture incidence is demonstrated in (Table 4). According to the cox regression models, NC group dis-

Table 4. Risk of vertebral fractures according to smoking status and history

\begin{tabular}{|c|c|c|c|c|c|c|c|}
\hline & $\mathrm{N}$ & $\begin{array}{c}\text { Vertebral } \\
\text { fractures } \\
\text { (total) }\end{array}$ & Duration* & $\mathrm{IR}^{\dagger}$ & $\begin{array}{c}\text { MODEL } 1 \\
\text { aHR (95\% CI) }\end{array}$ & $\begin{array}{c}\text { MODEL } 2 \\
\text { aHR (95\% CI) }\end{array}$ & $\begin{array}{c}\text { MODEL } 3 \\
\text { aHR (95\% CI) }\end{array}$ \\
\hline \multicolumn{8}{|l|}{$\mathrm{NC}$} \\
\hline Non & 530,503 & 15,763 & $1,842,451.36$ & 8.5554 & 1 (ref.) & 1 (ref.) & 1 (ref.) \\
\hline Ex & 126,503 & 1,878 & $424,404.43$ & 4.425 & $0.917(0.866,0.971)$ & $0.945(0.892,1.001)$ & $0.943(0.89,0.999)$ \\
\hline Current & 102,868 & 2,236 & $357,392.86$ & 6.2564 & $1.22(1.158,1.285)$ & $1.194(1.132,1.259)$ & $1.194(1.133,1.259)$ \\
\hline Non & 530,503 & 15,763 & $1,842,451.36$ & 8.5554 & 1 (ref.) & 1 (ref.) & 1 (ref.) \\
\hline $\mathrm{Ex} /<20$ py & 60,207 & 798 & $199,880.58$ & 3.9924 & $0.818(0.757,0.884)$ & $0.855(0.791,0.924)$ & $0.852(0.788,0.92)$ \\
\hline $\mathrm{Ex} / \geq 20 \mathrm{py}$ & 66,296 & 1,080 & $224,523.85$ & 4.8102 & $1.012(0.944,1.085)$ & $1.028(0.958,1.102)$ & $1.027(0.957,1.101)$ \\
\hline Current $/<20$ py & 32,885 & 760 & $112,345.62$ & 6.7648 & $1.205(1.117,1.3)$ & $1.201(1.113,1.296)$ & $1.201(1.113,1.296)$ \\
\hline Current/ $\geq 20$ py & 69,983 & 1,476 & $245,047.24$ & 6.0233 & $1.231(1.158,1.31)$ & $1.193(1.12,1.27)$ & $1.194(1.121,1.271)$ \\
\hline \multicolumn{8}{|l|}{ SCD } \\
\hline Non & 138,322 & 5,702 & $524,236.53$ & 10.8768 & 1 (ref.) & 1 (ref.) & 1 (ref.) \\
\hline Ex & 34,009 & 683 & $121,343.07$ & 5.6287 & $0.93(0.842,1.028)$ & $0.958(0.867,1.06)$ & $0.959(0.867,1.06)$ \\
\hline Current & 23,034 & 725 & $86,279.2$ & 8.403 & $1.283(1.169,1.408)$ & $1.257(1.143,1.381)$ & $1.267(1.153,1.393)$ \\
\hline Non & 138,322 & 5,702 & $524,236.53$ & 10.8768 & 1 (ref.) & 1 (ref.) & 1 (ref.) \\
\hline $\mathrm{Ex} /<20$ py & 15,380 & 285 & $53,891.96$ & 5.2884 & $0.845(0.741,0.965)$ & $0.881(0.772,1.006)$ & $0.88(0.771,1.005)$ \\
\hline $\mathrm{Ex} / \geq 20$ py & 18,629 & 398 & $67,451.1$ & 5.9006 & $1.005(0.89,1.134)$ & $1.02(0.903,1.153)$ & $1.022(0.905,1.155)$ \\
\hline Current/<20 py & 7,088 & 259 & $26,041.82$ & 9.9455 & $1.325(1.164,1.508)$ & $1.315(1.154,1.498)$ & $1.326(1.164,1.51)$ \\
\hline Current $/ \geq 20$ py & 15,946 & 466 & $60,237.38$ & 7.7361 & $1.263(1.128,1.414)$ & $1.225(1.092,1.374)$ & $1.235(1.101,1.386)$ \\
\hline \multicolumn{8}{|l|}{ Dementia } \\
\hline Non & 9,895 & 399 & $29,764.81$ & 13.4051 & 1 (ref.) & 1 (ref.) & 1 (ref.) \\
\hline Ex & 1,863 & 41 & $5,474.52$ & 7.4892 & $0.946(0.644,1.389)$ & $0.948(0.644,1.395)$ & $0.944(0.641,1.391)$ \\
\hline Current & 1,243 & 33 & $3,560.81$ & 9.2676 & $1.106(0.743,1.647)$ & $1.128(0.753,1.689)$ & $1.131(0.754,1.695)$ \\
\hline Non & 9,895 & 399 & $29,764.81$ & 13.4051 & 1 (ref.) & 1 (ref.) & 1 (ref.) \\
\hline $\mathrm{Ex} /<20 \mathrm{py}$ & 812 & 19 & $2,347.78$ & 8.0928 & $0.968(0.589,1.591)$ & $0.964(0.585,1.59)$ & $0.94(0.57,1.55)$ \\
\hline $\mathrm{Ex} / \geq 20 \mathrm{py}$ & 1,051 & 22 & $3,126.74$ & 7.0361 & $0.907(0.558,1.475)$ & $0.913(0.561,1.487)$ & $0.926(0.567,1.511)$ \\
\hline Current/<20 py & 527 & 18 & $1,491.28$ & 12.0702 & $1.324(0.808,2.169)$ & $1.332(0.81,2.19)$ & $1.358(0.825,2.233)$ \\
\hline Current $/ \geq 20$ py & 716 & 15 & $2,069.53$ & 7.248 & $0.906(0.52,1.581)$ & $0.931(0.529,1.638)$ & $0.921(0.524,1.62)$ \\
\hline
\end{tabular}

MODEL 1: Sex. MODEL 2: Sex, income, diabetes mellitus, hypertension, hyperlipidemia, alcohol drinking status, physical exercise, body mass index. MODEL 3: Sex, income, diabetes mellitus, hypertension, hyperlipidemia, alcohol drinking status, physical exercise, body mass index, fracture history. *Person-years, tincidence rate per 1,000. NC: normal cognition, SCD: subjective cognitive decline, py: pack years, aHR: adjusted hazard ratio, CI: confidence interval 
played increased risk of vertebral fractures in current smokers. ( $\mathrm{aHR}=1.194 ; 95 \% \mathrm{CI}=1.33-1.259)$, while decreased risk in ex-smokers ( $\mathrm{aHR}=0.943$; 95\% CI=0.89-0.999). Current smokers, regardless of pack years, were at increased risk of vertebral fractures. In SCD group, risk of vertebral fracture incidence increased in current smokers $(\mathrm{aHR}=1.267 ; 95 \% \mathrm{CI}=$ 1.153-1.393). The highest risk was observed in current smokers $<20$ py $(\mathrm{aHR}=1.326$; 95\% CI=1.164-1.51). No significant findings were observed in the dementia group.

\section{Risk of other fracture incidence}

Risk of other fractures incidence is demonstrated in (Table 5). In NC group, current smokers ( $\mathrm{aHR}=1.08$; 95\% $\mathrm{CI}=1.022-$ $1.143)$, current smokers with $<20$ py $(\mathrm{aHR}=1.122 ; 95 \% \mathrm{CI}=$
1.037-1.214) were all concordantly at increased risk of other fractures. No significant findings were observed in SCD or dementia group.

\section{DISCUSSION}

We aimed to demonstrate the risk of fractures in SCD and dementia group with regard to the smoking status of participants. To our knowledge, this is the first study to elucidate the differential impact of smoking on increasing fracture risk in SCD and dementia groups. In NC and SCD group, when compared with ex-smokers, current smoking concordantly increased all, hip, vertebral fractures with higher risk, even with consideration for history of heavy smoking ( $\geq 20$ py).

Table 5. Risk of other fractures according to smoking status and history

\begin{tabular}{|c|c|c|c|c|c|c|c|}
\hline & $\mathrm{N}$ & $\begin{array}{c}\text { Other fractures } \\
\text { (total) }\end{array}$ & Duration* & $\mathrm{IR}^{\dagger}$ & $\begin{array}{c}\text { MODEL } 1 \\
\text { aHR (95\% CI) }\end{array}$ & $\begin{array}{c}\text { MODEL } 2 \\
\text { aHR (95\% CI) }\end{array}$ & $\begin{array}{c}\text { MODEL } 3 \\
\text { aHR (95\% CI) }\end{array}$ \\
\hline \multicolumn{8}{|l|}{$\mathrm{NC}$} \\
\hline Non & 530,503 & 16,460 & $1,842,451.36$ & 8.9338 & 1 (ref.) & 1 (ref.) & 1 (ref.) \\
\hline Ex & 126,503 & 1,895 & $424,404.43$ & 4.4651 & $0.997(0.942,1.056)$ & $0.986(0.931,1.045)$ & $0.984(0.928,1.043)$ \\
\hline Current & 102,868 & 1,936 & $357,392.86$ & 5.417 & $1.128(1.068,1.192$ & $1.08(1.021,1.142)$ & $1.08(1.022,1.143)$ \\
\hline Non & 530,503 & 16,460 & $1,842,451.36$ & 8.9338 & 1 (ref.) & 1 (ref.) & 1 (ref.) \\
\hline $\mathrm{Ex} /<20$ py & 60,207 & 870 & $199,880.58$ & 4.3526 & $0.952(0.883,1.026)$ & $0.948(0.879,1.022)$ & $0.944(0.876,1.018)$ \\
\hline $\mathrm{Ex} / \geq 20 \mathrm{py}$ & 66,296 & 1,025 & $224,523.85$ & 4.5652 & $1.038(0.966,1.115)$ & $1.018(0.947,1.094)$ & $1.017(0.946,1.093)$ \\
\hline Current/ $<20$ py & 32,885 & 704 & $112,345.62$ & 6.2664 & $1.157(1.07,1.251)$ & $1.121(1.036,1.213)$ & $1.122(1.037,1.214)$ \\
\hline Current $/ \geq 20$ py & 69,983 & 1,232 & $245,047.24$ & 5.0276 & $1.112(1.041,1.188)$ & $1.055(0.986,1.129)$ & $1.056(0.987,1.129)$ \\
\hline \multicolumn{8}{|l|}{ SCD } \\
\hline Non & 138,322 & 5,478 & $524,236.53$ & 10.4495 & 1 (ref.) & 1 (ref.) & 1 (ref.) \\
\hline Ex & 34,009 & 679 & $121,343.07$ & 5.5957 & $1.032(0.931,1.143)$ & $1.017(0.917,1.128)$ & $1.017(0.916,1.128)$ \\
\hline Current & 23,034 & 542 & $86,279.2$ & 6.2819 & $1.068(0.963,1.185)$ & $1.009(0.908,1.121)$ & $1.018(0.916,1.131)$ \\
\hline Non & 138,322 & 5,478 & $524,236.53$ & 10.4495 & 1 (ref.) & 1 (ref.) & 1 (ref.) \\
\hline $\mathrm{Ex} /<20$ py & 15,380 & 314 & $53,891.96$ & 5.8265 & $1.031(0.907,1.173)$ & $1.025(0.901,1.166)$ & $1.023(0.899,1.165)$ \\
\hline $\mathrm{Ex} / \geq 20$ py & 18,629 & 365 & $67,451.1$ & 5.4113 & $1.04(0.915,1.181)$ & $1.013(0.891,1.152)$ & $1.015(0.892,1.154)$ \\
\hline Current $/<20$ py & 7,088 & 185 & $26,041.82$ & 7.104 & $1.029(0.884,1.196)$ & $0.988(0.849,1.15)$ & $0.996(0.856,1.16)$ \\
\hline Current $/ \geq 20$ py & 15,946 & 357 & $60,237.38$ & 5.9266 & $1.095(0.966,1.242)$ & $1.022(0.899,1.162)$ & $1.032(0.908,1.173)$ \\
\hline \multicolumn{8}{|l|}{ Dementia } \\
\hline Non & 9,895 & 332 & $29,764.81$ & 11.1541 & 1 (ref.) & 1 (ref.) & 1 (ref.) \\
\hline Ex & 1,863 & 34 & $5,474.52$ & 6.2106 & $0.943(0.618,1.439)$ & $0.893(0.584,1.367)$ & $0.895(0.584,1.371)$ \\
\hline Current & 1,243 & 24 & $3,560.81$ & 6.74 & $0.961(0.607,1.521)$ & $0.87(0.544,1.391)$ & $0.866(0.541,1.386)$ \\
\hline Non & 9,895 & 332 & $29,764.81$ & 11.1541 & 1 (ref.) & 1 (ref.) & 1 (ref.) \\
\hline $\mathrm{Ex} /<20$ py & 812 & 16 & $2,347.78$ & 6.815 & $0.997(0.579,1.718)$ & $0.954(0.553,1.647)$ & $0.936(0.542,1.617)$ \\
\hline $\mathrm{Ex} / \geq 20$ py & 1,051 & 18 & $3,126.74$ & 5.7568 & $0.909(0.531,1.557)$ & $0.85(0.495,1.458)$ & $0.866(0.504,1.488)$ \\
\hline Current/<20 py & 527 & 9 & $1,491.28$ & 6.0351 & $0.801(0.405,1.582)$ & $0.781(0.394,1.548)$ & $0.788(0.397,1.561)$ \\
\hline Current $/ \geq 20$ py & 716 & 15 & $2,069.53$ & 7.248 & $1.102(0.625,1.944)$ & $0.939(0.525,1.679)$ & $0.926(0.518,1.655)$ \\
\hline
\end{tabular}

MODEL 1: Sex, MODEL 2: Sex, income, diabetes mellitus, hypertension, hyperlipidemia, alcohol drinking status, physical exercise, body mass index, MODEL 3: Sex, income, diabetes mellitus, hypertension, hyperlipidemia, alcohol drinking status, physical exercise, body mass index, fracture history. *Person-years, tincidence rate per 1,000. NC: normal cognition, SCD: subjective cognitive decline, py: pack years, aHR: adjusted hazard ratio, CI: confidence interval 
SCD group was more vulnerable than NC group, displaying higher hazard ratios in current smokers. In contrast, risk of hip fractures in current smokers $\geq 20$ py was reduced in dementia group. The aforementioned findings all convey several important clinical implications.

First, increased risk of all, hip, vertebral fractures in current smokers regardless of the pack year status, in both NC and SCD group alike, highlight the well-known deleterious effects of smoking. Current smoking itself, regardless of pack years, might exert detrimental influence on bone heath of the elderly. With regard to the link between fractures and smoking, numerous research has reported the deleterious effect of smoking on bones and muscles, which ultimately leads to increased fracture risks Smoking was associated with reduced bone mineral density, cortical volume, ${ }^{25}$ and bone mass. ${ }^{26} \mathrm{Cal}-$ cium intake was reduced by smoking, ${ }^{27}$ and smoking detrimentally affected mRNA expression of bone-related markers. ${ }^{28}$ In a recent study, pernicious effects of smoking on bone mineralization and collagen synthesis were affected in a mouse model, demonstrating that mice exposed to cigarette smoke exhibited reduction of type I collagen deposition, which is critical for bone formation, which ultimately lead to slowed delayed bone formation rate. ${ }^{29}$ Moreover, smoking was an independent predictor of worse trabecular strength in hip fracture patients. ${ }^{30}$ As for the indirect effect of smoking on bone health, smoking was associated with decreased levels of parathyroid hormone ${ }^{31}$ and vitamin $\mathrm{D},{ }^{32}$ which could undermine bone integrity.

Second, in NC group, there were marginal reduction of hazard ratios in ex-smokers with regard to all and vertebral fractures. Dose-dependent, duration-dependent increase of fracture risks in smokers has previously been reported, with prediction of ex-smokers at intermediate fracture risk between that of never smokers and current smokers. ${ }^{21}$ In this regard, smoking cessation might help reduce fracture risks to some extent. A contrasting result exists, where 75-year old elderly women were followed up for 10 years, reporting no evidence of smoking cessation reducing fracture risks except for vertebral fractures. ${ }^{17}$ This study also demonstrated that the amount of smoked cigarettes did not influence the risk. ${ }^{17} \mathrm{Nev}$ ertheless, the importance of smoking cessation should not be underrated, since subjects of our study were not asked for the duration of smoking cessation while cessation of smoking for more than 10 years was reported to be protective for hip fractures in women, ${ }^{33}$ and as long as 30 years in men. ${ }^{34}$

Third, higher risks of all, hip, vertebral fractures were noted in current smokers in SCD group when compared with smokers in NC group. Subjects with SCD were clearly at higher risk of fractures than that of NC group. Evidences on risks of falls or fractures in SCD are scarce. Previous studies report- ed increased risk of falls and hip fractures in SCD group. ${ }^{12,15}$ The reason for this increase in risk of falls or fracture in SCD could be attributed to additive role of frailty on harmful effects of smoking. Frailty was closely associated with SCD long before manifestation of objective cognitive impairment. ${ }^{35}$ Especially, subjective complaints in mathematic reasoning were highly associated with frailty. ${ }^{36}$ Smokers in SCD group in our study was likely to have been exposed to increased chance of frailty than their NC counterpart. Increasing literatures are beginning to focus on SCD with physical frailty, reversible cognitive frailty, ${ }^{37,38}$ and unraveling its relationship with fracture and dementia risks will be a novel arena of research.

Fourth, risk of hip fractures in current smokers were reduced in dementia group, when compared with SCD and NC group. One can easily assume that fracture risks will be increased proportionally with severity of cognitive impairment in dementia, but studies report otherwise results. Compared to those with mild dementia, those with moderate dementia was exposed to lower risk of hip fractures in a Korean nationwide longitudinal study. ${ }^{4}$ The study elucidated the reasons for such finding can be attributable to decreased outside activity, loss of independence and depressive symptoms. ${ }^{4}$ Another study called for the need for a different perspective when assessing fracture risks in Alzheimer's disease (AD) patients, suggesting that the influence of $\mathrm{AD}$ per se, associated medication (e.g. psychotropic medication) or environment can overrule the effects of other important risk factors of hip fractures. ${ }^{39}$ Another significant factor that might have influenced our results is decreased prevalence of current and ex-smokers in dementia group. Prevalence of smokers were significantly reduced in dementia group when compared with subjects with normal cognition, and suggested reasons were early changes of nicotinic receptors before $\mathrm{AD}$ pathology, with resultant loss of craving for smoking. ${ }^{40}$ Significantly lower rates of current smokers were found in dementia patients, suggesting two following reasons as possible explanations: 1) forgetting that they were smokers and how to smoke, 2) increased supervision by caregivers. ${ }^{41}$ Nicotinic receptors are another domain that must be considered for interpretation of our results in dementia group. Beneficial effects of nicotine on improving attention, and positive modulation of nicotinic receptors were proposed to be protective against amyloid beta toxicity. ${ }^{42}$ In this regard, for subjects with dementia, nicotine exposure might have provided enhanced cognitive performance that could have reduced the fracture risks. This could explain a slightly deviant result on the increased risk of all, vertebral, hip fractures in current smokers $<20$ py when compared with current smokers $\geq 20$ py. In current smokers with dementia, increased nicotine dose might exert protective effect against fractures. Lastly, effects of AchEI should be taken into account. Use of 
beta-blockers inhibited sympathetic activity, and thereby increasing bone density. ${ }^{43}$ Muscarinic activity of AchEI could have activated parasympathetic system that enhance bone formation. ${ }^{44}$ In dementia patients, beneficial effects of AChEI on bone formation could have counteracted the toxic effects of nicotine exposure on bones.

There are several limitations that must be taken into consideration with regard to our study results. First, classification of SCD might not reflect the pure clinical population that is devoid of objective cognitive impairment, since the study did not include any objective measurements of cognition. However, according to one study on patients with $\mathrm{SCD}$, subjective awareness of one's cognitive decline was decreased in amnestic mild cognitive impairment (aMCI) and AD patients. ${ }^{45}$ By exclusively selecting subjects with KDSQ-P scores of 4 or more, those with higher subjective awareness of cognitive decline, there was an increased chance we excluded those with objective cognitive impairments. Second, definition of dementia was solely based on the reimbursement criteria, which did not include objective cognitive tests or neuroimaging results. However, we believe that the relative strict reimbursement criteria, a mandatory submission of test results on cognitive scores and functional assessment scores, increased the chance of inclusion of dementia patients. Third, data on common risk factors of fractures in dementia patients, including prescription of psychotropic medication were not provided in the study. Fourth, additional adjustment for factors such as activities of daily living, fall history, BMD, use of corticosteroids, bisphosphonate prescription history, sensory impairments in later life ${ }^{46}$ could have further strengthened our results on the link between fractures and subjects with cognitive impairment. Fifth, since the data on smoking status was collected through surveys, it was subject to recall bias. ${ }^{47}$ Sixth, since the cohort was 66 years old at the time of screening, those on dementia medication at the time of screening are likely to be reflect early onset dementia patients, which might not reflect the actual clinical late onset dementia patients we often encounter in clinics. Anyhow, the strength of our study lies on the unprecedented big, real-world data on smokers with SCD or dementia, and adjustment for many variables that could affect fracture risks.

In conclusion, fracture risks were elevated in current heavy smokers in SCD, but not in dementia. Future studies should focus on unraveling the causes of this differential impact of cigarette smoking on fracture risks of SCD and dementia groups. Fracture risks are still under-evaluated and undertreated in memory clinics. ${ }^{48} \mathrm{SCD}$ was closely related to subjective awareness of physical function, ${ }^{49}$ which warrant the importance early detection and assessment of physical deterioration and fracture risks in the early stages of cognitive im- pairment. Moreover, personalized preventive measures according to one's cognitive status are imperative, since risk factors of fractures can exert disparate influence on patients at different stage of cognitive trajectory.

\section{Acknowledgments}

This work was supported by the National Research Foundation of Korea (NRF) grant funded by the Korea government (MSIT) (No. 2019R1A2 C2009100).

\section{Conflicts of Interest}

The authors have no potential conflicts of interest to disclose.

\section{Author Contributions}

Conceptualization: Yoo Hyun Um, Hyun Kook Lim. Data curation: Hyun Kook Lim. Formal analysis: Hyun Kook Lim. Funding acquisition: Hyun Kook Lim. Investigation: Sheng-Min Wang, Dong Woo Kang, NakYoung Kim, Hae-Ran Na. Methodology: Kyung-do Han. Project administration: Kyung-do Han, Hyun Kook Lim. Resources: Hyun Kook Lim. Supervision: Chang Uk Lee. Writing_original draft: Yoo Hyun Um. Writingreview \& editing: Sheng-Min Wang, Dong Woo Kang, Nak-Young Kim, Hae-Ran Na, Kyung-do Han.

\section{ORCID iDs}

Yoo Hyun Um

Sheng-Min Wang

Kyung-do Han

Nak-Young Kim

Dong Woo Kang

Hae-Ran $\mathrm{Na}$

Chang Uk Lee

https://orcid.org/0000-0002-3403-4140

https://orcid.org/0000-0003-2521-1413 https://orcid.org/0000-0002-6096-1263 https://orcid.org/0000-0003-0116-6283 https://orcid.org/0000-0003-3289-075X https://orcid.org/0000-0002-7960-8603

Hyun Kook Lim https://orcid.org/0000-0001-6398-7330 https://orcid.org/0000-0001-8742-3409

\section{REFERENCES}

1. Feil D, Marmon T, Unützer J. Cognitive impairment, chronic medical illness, and risk of mortality in an elderly cohort. Am J Geriatr Psychiatry 2003;11:551-560.

2. Gruber-Baldini AL, Zimmerman S, Morrison RS, Grattan LM, Hebel JR, Dolan MM, et al. Cognitive impairment in hip fracture patients: timing of detection and longitudinal follow-up. J Am Geriatr Soc 2003; 51:1227-1236.

3. Pareja Sierra T, Bartolomé Martín I, Rodríguez Solís J, Bárcena Goitiandia L, Torralba González de Suso M, Morales Sanz, et al. Predictive factors of hospital stay, mortality and functional recovery after surgery for hip fracture in elderly patients. Rev Esp Cir Ortop Traumatol 2017; 61:427-435.

4. Jeon JH, Park JH, Oh C, Chung JK, Song JY, Kim S, et al. Dementia is associated with an increased risk of hip fractures: a nationwide analysis in Korea. J Clin Neurol 2019;15:243-249.

5. Liang Y, Wang L. Alzheimer's disease is an important risk factor of fractures: a meta-analysis of cohort studies. Mol Neurobiol 2017;54: 3230-3235.

6. Wang HK, Hung CM, Lin SH, Tai YC, Lu K, Liliang PC, et al. Increased risk of hip fractures in patients with dementia: a nationwide population-based study. BMC Neurol 2014;14:175-175.

7. Gras LZ, Kanaan SF, McDowd JM, Colgrove YM, Burns J, Pohl PS. Balance and gait of adults with very mild Alzheimer disease. J Geriatr Phys Ther 2015;38:1-7.

8. Ogawa Y, Kaneko Y, Sato T, Shimizu S, Kanetaka H, Hanyu H. Sarcopenia and muscle functions at various stages of Alzheimer disease. Front Neurol 2018;9:710-710.

9. Ruan Q, Yu Z, Chen M, Bao Z, Li J, He W. Cognitive frailty, a novel 
target for the prevention of elderly dependency. Ageing Res Rev 2015; 20:1-10.

10. Rabin LA, Smart CM, Amariglio RE. Subjective cognitive decline in preclinical Alzheimer's disease. Annu Rev Clin Psychol 2017;13:369-396.

11. Buckley RF, Saling MM, Frommann I, Wolfsgruber S, Wagner M. Subjective cognitive decline from a phenomenological perspective: a review of the qualitative literature. J Alzheimers Dis 2015;48 (Suppl 1): S125-S140.

12. Shirooka H, Nishiguchi S, Fukutani N, Tashiro Y, Nozaki Y, Aoyama T. Subjective cognitive decline and fall risk in community-dwelling older adults with or without objective cognitive decline. Aging Clin Exp Res 2018:30:457-462.

13. Hsieh TJ, Chang HY, Wu IC, Chen CC, Tsai HJ, Chiu YF, et al. Independent association between subjective cognitive decline and frailty in the elderly. PLoS One 2018;13:e0201351.

14. Al-Sari UA, Tobias JH, Archer H, Clark EM. Do subjective memory complaints predict falls, fractures and healthcare utilization? A two-year prospective study based on a cohort of older women recruited from primary care. Int J Geriatr Psychiatry 2017;32:968-976.

15. Garcia Lopez M, Omsland TK, Sogaard AJ, Meyer HE. Self-perceived memory loss is associated with an increased risk of hip fracture in the elderly: a population-based NOREPOS cohort study. BMC Geriatr 2015;15:134

16. Organization WH. WHO Global Report on Trends in Prevalence of Tobacco Smoking 2000-2025. Geneva: World Health Organization; 2018.

17. Thorin MH, Wihlborg A, Åkesson K, Gerdhem P. Smoking, smoking cessation, and fracture risk in elderly women followed for 10 years. Osteoporos Int 2016;27:249-255.

18. Wiklund R, Toots A, Conradsson M, Olofsson B, Holmberg H, Rosendahl E, et al. Risk factors for hip fracture in very old people: a population-based study. Osteoporos Int 2016;27:923-931.

19. Jutberger H, Lorentzon M, Barrett-Connor E, Johansson H, Kanis JA, Ljunggren $\mathrm{O}$, et al. Smoking predicts incident fractures in elderly men: Mr OS Sweden. J Bone Miner Res 2010;25:1010-1016.

20. Lee JJ, Patel R, Biermann JS, Dougherty PJ. The musculoskeletal effects of cigarette smoking. J Bone Joint Surg Am 2013;95:850-859.

21. Yoon V, Maalouf NM, Sakhaee K. The effects of smoking on bone metabolism. Osteoporos Int 2012;2:2081-2092.

22. Kim HS, Shin DW, Lee WC, Kim YT, Cho B. National screening program for transitional ages in Korea: a new screening for strengthening primary prevention and follow-up care. J Korean Med Sci 2012;27(Suppl):S70-S75.

23. Cheol Seong S, Kim YY, Khang YH, Heon Park J, Kang HJ, Lee H, et al. Data resource profile: the National health information database of the National health insurance service in South Korea. Int J Epidemiol 2017;46:799-800.

24. Jeon Y, Yun K, Kim Y. Validation of KDSQ-P as selecting elderly for KDSQ-C. Korean J Health Promot 2010;10:45-52.

25. Marques EA, Elbejjani M, Gudnason V, Sigurdsson G, Lang T, Sigurdsson S, et al. Cigarette smoking and hip volumetric bone mineral density and cortical volume loss in older adults: the AGES-Reykjavik study. Bone 2018;108:186-192.

26. Callréus M, McGuigan F, Akesson K. Adverse effects of smoking on peak bone mass may be attenuated by higher body mass index in young female smokers. Calcif Tissue Int 2013;93:517-525.

27. Breitling LP. Smoking as an effect modifier of the association of calcium intake with bone mineral density. J Clin Endocrinol Metab 2015;100: 626-635.

28. Campos JM, Prati AJ, Cirano FR, Pimentel SP, Pastore GP, Pecorari $\mathrm{VG}$, et al. Smoking modulates gene expression of type I collagen, bone sialoprotein, and osteocalcin in human alveolar bone. J Oral Maxillofac Surg 2015;73:2123-2131.

29. Barbosa AP, Lourenço JD, Junqueira JJM, Larissa Emidio de França S, Martins JS, Oliveira Junior MC, et al. The deleterious effects of smoking in bone mineralization and fibrillar matrix composition. Life Sci
2020;241:117132.

30. Rodrigues AM, Caetano-Lopes J, Vale AC, Fonseca JE, Canhão H. Smoking is a predictor of worse trabecular mechanical performance in hip fragility fracture patients. J Bone Miner Metab 2012;30:692-699.

31. Fujiyoshi A, Polgreen LE, Gross MD, Reis JP, Sidney S, Jacobs DR Jr. Smoking habits and parathyroid hormone concentrations in young adults: the CARDIA study. Bone Reports 2016;5:104-109.

32. Kassi EN, Stavropoulos S, Kokkoris P, Galanos A, Moutsatsou P, Dimas C, et al. Smoking is a significant determinant of low serum vitamin D in young and middle-aged healthy males. Hormones (Athens) 2015;14: 245-250.

33. Shen GS, Li Y, Zhao G, Zhou HB, Xie ZG, Xu W, et al. Cigarette smoking and risk of hip fracture in women: a meta-analysis of prospective cohort studies. Injury 2015;46:1333-1340.

34. Olofsson H, Byberg L, Mohsen R, Melhus H, Lithell H, Michaëlsson K. Smoking and the risk of fracture in older men. J Bone Miner Res 2005;20: 1208-1215.

35. Gifford KA, Bell SP, Liu D, Neal JE, Turchan M, Shah AS, et al. Frailty is related to subjective cognitive decline in older women without dementia. J Am Geriatr Soc 2019;67:1803-1811.

36. Margioti E, Kosmidis MH, Yannakoulia M, Dardiotis E, Hadjigeorgiou G, Sakka P, et al. Exploring the association between subjective cognitive decline and frailty: the Hellenic Longitudinal Investigation of Aging and Diet Study (HELIAD). Aging Ment Health 2020;24:137-147.

37. Solfrizzi V, Scafato E, Seripa D, Lozupone M, Imbimbo BP, D’Amato A, et al. Reversible cognitive frailty, dementia, and all-cause mortality. The Italian longitudinal study on aging. J Am Med Dir Assoc 2017;18:89.e8189.e88.

38. Panza F, Lozupone M, Solfrizzi V, Sardone R, Dibello V, Di Lena L, et al. Different cognitive frailty models and health- and cognitive-related outcomes in older age: from epidemiology to prevention. J Alzheimers Dis 2018;62:993-1012.

39. Tolppanen AM, Taipale H, Tanskanen A, Tiihonen J, Hartikainen S. Comparison of predictors of hip fracture and mortality after hip fracture in community-dwellers with and without Alzheimer's disease-exposure-matched cohort study. BMC Geriatr 2016;16:204.

40. Barclay L, Kheyfets S. Tobacco use in Alzheimer's disease. Prog Clin Biol Res 1989;317:189-194.

41. Lester PE, Lyubarova R, Kirtani V, Macina LO, Kohen I. Smoking rates in dementia patients in the outpatient setting. Arch Gerontol Geriatr 2011;52:281-283.

42. Echeverria V, Zeitlin R. Cotinine: a potential new therapeutic agent against Alzheimer's disease. CNS Neurosci Ther 2012;18:517-523.

43. Schlienger RG, Kraenzlin ME, Jick SS, Meier CR. Use of beta-blockers and risk of fractures. JAMA 2004;292:1326-1332.

44. Tamimi I, Ojea T, Sanchez-Siles JM, Rojas F, Martin I, Gormaz I, et al. Acetylcholinesterase inhibitors and the risk of hip fracture in Alzheimer's disease patients: a case-control study. J Bone Miner Res 2012;27: 1518-1527.

45. Lehrner J, Kogler S, Lamm C, Moser D, Klug S, Pusswald G, et al. Awareness of memory deficits in subjective cognitive decline, mild cognitive impairment, Alzheimer's disease and Parkinson's disease. Int Psychogeriatr 2015;27:357-366.

46. Lyu J, Kim HY. Gender-specific associations of sensory impairments with depression and cognitive impairment in later life. Psychiatry Investig 2018;15:926-934.

47. Krall EA, Valadian I, Dwyer JT, Gardner J. Accuracy of recalled smoking data. Am J Public Health 1989;79:200-202.

48. Lampshire Z, Tingley D, Jarvis A, Wernham C, Hughes JC, Welsh TJ. Fracture risk is under-recognised and under-treated in memory clinic attendees. Maturitas 2019;123:37-39.

49. Cosentino S, Devanand D, Gurland B. A link between subjective perceptions of memory and physical function: implications for subjective cognitive decline. J Alzheimers Dis 2018;61:1387-1398. 